\title{
Fully Integrated SOI Wavelength Meter Based on Phase Shift Technique
}

\author{
Alfonso Ruocco and Wim Bogaerts \\ Photonics Research Group, Ghent University - imec, Department of Information Technology \\ Center of Nano- and Biophotonics (NB-photonics), Ghent, Belgium \\ Email: Alfonso.Ruocco@intec.UGent.be
}

\begin{abstract}
We present an MZI (Mach-Zehnder Interferometer) silicon-on-insulator (SOI) wavelength meter. The device integrates photo detectors and modulators. The phase shift between carrier and modulated signal is linearly correlated to the input wavelength.
\end{abstract}

\section{INTRODUCTION}

Many applications require compact, reliable and accurate wavelength metering of one or more peaks. These include (bio)sensors, fiber Bragg grating readouts, or tunable laser sources. The most used solutions such as wavelength filters suffer from limitations due to fabrication tolerances and variability. Other approaches required movable parts or a complex PIC (photonic integrated circuit) [1]. We propose an elegant solution using a single asymmetric MZI, with high speed interdigitated carrier depletion modulators are integrated in each arm. On the output Germanium photodiodes (PD) convert the optical power into an electrical signal. The technology platform is based on silicon-on-insulator of $220 \mathrm{~nm}$. The principle used for the wavelength recovery relies on the sampling properties of an ideal Dirac pulse. The operating wavelength range of our device is equal to half of the FSR of the MZI, which is $10 \mathrm{~nm}$, but this can be extended to the full FSR. The measured worst-case accuracy is $144 \mathrm{pm}$ with an average error of $73 \mathrm{pm}$. The footprint is mainly limited by the size of the modulators, but it can be significantly reduced. We used carrier-based modulators, but it is also possible to use thermo-optic modulators, which are more efficient, compact and have a lower insertion loss.

\section{WORKING PRINCIPLE}

The wavelength meter consists of an asymmetric MZI. The splitting and combining is realized with $2 \times 2 \mathrm{MMI}$ (Multimode Interferometers) couplers: this device offers good wavelength and processing tolerances when a $3 d B$ splitting ratio is required. The delay lines are realized with standard SOI wire waveguides of $450 \mathrm{~nm}$ with $800 \mathrm{~nm}$ straight sections. The modulators consist of $150 \mathrm{~nm}$ etched waveguides and interdigitated $p n$-junctions [2]. The length of the active sections is $1.5 \mathrm{~mm}$, but long folded twice such that the overall length of the component is only $0.5 \mathrm{~mm}$. The outputs of the MZI are connected to Germanium PDs [3]. Grating couplers are used for the input of the circuit. Fig 1 shows an image of the device in which the main parts have been indicated and further magnified.

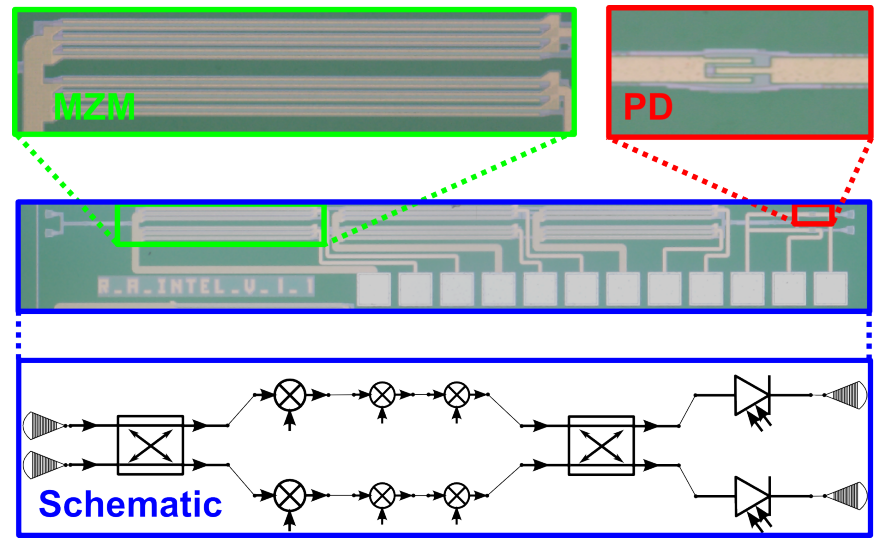

Fig. 1: Microscope image and schematic of the wavelength meter

Phase-shifting techniques are widely used for metrology purposes [4], and in this work we apply it to wavelength monitoring. The wavelength response of an MZI is a sinusoidal curve, and the exact position in the wavelength domain depends on the phase delay difference between the two arms. Eq. 1 describes the analytical relation of the MZI wavelength response assuming that the splitting ratio of the MMIs is an ideal 50/50 split, and that the losses in both arms are identical.

$$
T(\lambda)=\frac{1}{2}+\frac{1}{2} \sin \left(2 \pi n_{e f f}(\lambda) \frac{\Delta L}{\lambda}\right)
$$

The ideal input spectrum in the case of a wavelength meter is assumed to be a single wavelength peak which can be approximated by a Dirac pulse. Such simplification is in most of the cases reliable if the bandwidth of the peak is significantly smaller than the accuracy of the wavelength analyzer.The outputs of the MZI are connected to a PD. Therefore, the readout signal is an integral over all wavelengths. However, when assuming the input linewidth to be close to a Dirac impulse, the integral is equivalent to the MZI transmission at the peak wavelength, as expressed in eq. 1 evaluated at the wavelength of interest. When we modulate in the time domain the $n_{e f f}$ in one of the MZI arms, we actively shift the sinusoidal response of the device with a certain time-wavelength relation. We can then rewrite eq. 1 as

$$
V(t)=V_{d c}+V_{a c} \sin (\sin (\omega t)+\Delta \phi)
$$




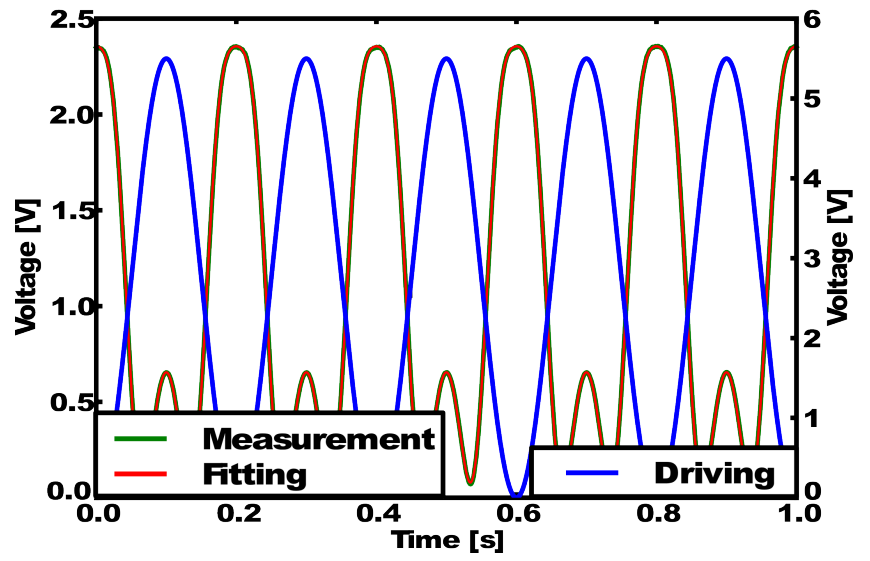

Fig. 2: Time domain driving, measurements and fitting@ $1540.0 \mathrm{~nm}$

The output at the photodetector becomes a modulated signal, converting the sine-like MZI wavelength response to an electrical time-domain response. The electrical phase term $\Delta \phi$ has a direct relationship with the peak wavelength:

$$
\Delta \phi=\frac{\left(\lambda_{r e s}-\lambda_{i n}\right)}{F S R}
$$

\section{Characterization of the PIC}

We characterized the PIC by sending in light from a tunable laser and measure the outputs of the photodetectors while modulating the MZI. A low noise TIA (trans-impedance amplifier) converts the photocurrent of the PD into a voltage. We also included access waveguides to calibrate the individual grating couplers, modulators and photodetectors in the circuit.

Fig. 2 represents the key measurements for an input wavelength of $1540.00 \mathrm{~nm}$. The blue curve (right axis) represents the driving signal: a sinusoidal curve with an offset (to avoid carrier injection in our PN junction). The green and red curves (left axis) represent the measured readout, as well as a fitting of the measurement onto a circuit model of the device. Since the model describes properly the time domain output, the fitting has a reduced quadratic error for all the wavelengths.

\section{WAVELENGTH MEASUREMENTS}

The device allows for different driving schemes. In this experimental phase, the response of the depletion MZI modulator is assumed to the linear with the applied reverse voltage. With this assumption, a sinusoidal waveform is used. The fitting between the measured signal and carrier provides noise cancellation. Also, instead of fitting to a sinusoidal curve, it is also possible to fit directly to a circuit model which includes more elaborate models for the building blocks.

More elaborate drive schemes can also help calibrate for modulator imperfections, imbalances, or for the simultaneous detection of multiple peaks in a spectrum.

$$
\Delta \lambda=\frac{F S R \Delta \phi}{2 \pi}
$$

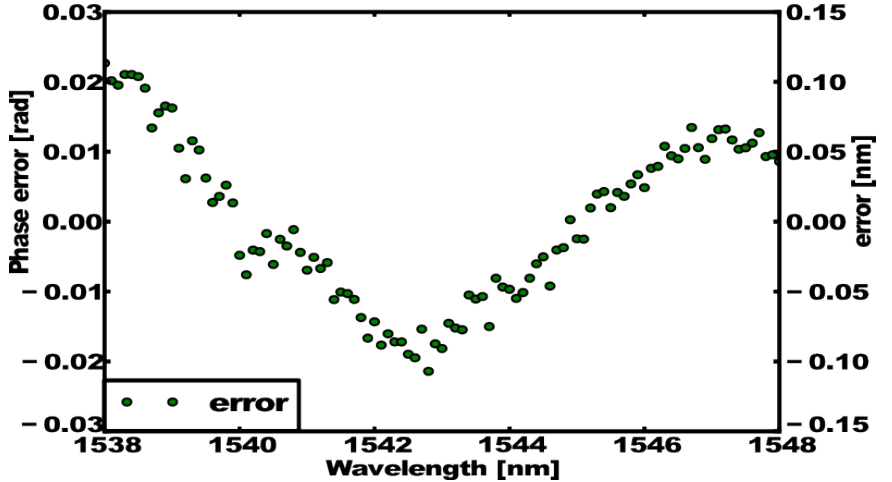

Fig. 3: Measured phase and wavelength error versus input wavelength

Fig. 3 shows the results of the wavelength measurements expressed as radians shift between carrier signal and output electrical signal. The right axe represent the conversion between radian error and wavelength accuracy correlated according to eq. 4. The plot represents half of the FSR. The curves belonging respectively to the electrical driving and to the electrical read-out are used for phase shift estimation. The driving signal is the reference and thus its phase is assumed to be zero. The readout signal is fitted with the eq. 2 and then the phase $\Delta \phi$ is converted to input wavelength relative to MZI resonance in the FSR of interest.

\section{CONCLUSIONS}

We demonstrated a MZI modulator used as a wavelength meter. We reported $73 \mathrm{pm}$ RMS accuracy on a operative range of $10 \mathrm{~nm}$. Future exploration include but are not limited different driving carriers, homodyne detection and on-chip phase detection.

\section{ACKNOWLEDGEMENT}

The simulation and design have been carried out with Luceda Software. The silicon PIC was manufactured through the IMEC MPW fabrication service.

\section{REFERENCES}

[1] A. Ruocco, D. Van Thourhout, and W. Bogaerts, "Silicon photonic spectrometer for accurate peak detection using the vernier effect and timedomain multiplexing," Lightwave Technology, Journal of, vol. 32, no. 19, pp. 3351-3357, Oct 2014.

[2] H. Yu, M. Pantouvaki, J. V. Campenhout et al., "Performance tradeoff between lateral and interdigitated doping patterns for high speed carrier-depletion based silicon modulators," Opt. Express, vol. 20, no. 12, pp. 12926-12938, Jun 2012. [Online]. Available: http://www.opticsexpress.org/abstract.cfm?URI=oe-20-12-12926

[3] P. Verheyen, G. Lepage, J. Van Campenhout et al., "Cointegration of ge detectors and si modulators in an advanced si photonics platform," pp. $843114-843114-8,2012$. [Online]. Available: http://dx.doi.org/10.1117/12.921747

[4] B. Costa, D. Mazzoni, M. Puleo et al., "Phase shift technique for the measurement of chromatic dispersion in optical fibers using led's," Quantum Electronics, IEEE Journal of, vol. 18, no. 10, pp. 1509-1515, Oct 1982 . 